\title{
Indian Hedgehog is an antagonist of Wnt signaling in colonic epithelial cell differentiation
}

\author{
Gijs R van den Brink ${ }^{1,6}$, Sylvia A Bleuming ${ }^{1,6}$, James C H Hardwick ${ }^{1}$, Berber L Schepman ${ }^{1}$, G Johan Offerhaus ${ }^{2}$, \\ Josbert J Keller ${ }^{2}$, Corinne Nielsen ${ }^{3}$, William Gaffield ${ }^{4}$, Sander J H van Deventer ${ }^{5}$, Drucilla J Roberts ${ }^{3}$ \\ \& Maikel P Peppelenbosch ${ }^{1}$
}

Wnt signaling defines the colonic epithelial progenitor cell phenotype $^{1}$, and mutations in the gene adenomatous polyposis coli $(A P C)$ that activate the Wnt pathway cause the familial adenomatous polyposis coli (FAP) syndrome and most sporadic colon cancers ${ }^{2}$. The mechanisms that regulate the transition of epithelial precursor cells into their differentiated derivatives are poorly characterized. We report that Indian hedgehog (Ihh) is expressed by mature colonocytes and regulates their differentiation in vitro and in vivo. Hedgehog $(\mathrm{Hh})$ signaling restricts the expression of Wnt targets to the base of the colonic crypt in vivo, and transfection of Ihh into colon cancer cells leads to a downregulation of both components of the nuclear TCF4- $\beta$-catenin complex and abrogates endogenous Wnt signaling in vitro. In turn, expression of Ihh is downregulated in polyps of individuals with FAP and expression of doxycyclineinducible dominant negative TCF4 (dnTCF4) restores Ihh expression in APC mutant DLD-1 colon cancer cells. These data identify a new Wnt-Hh axis in colonic epithelial renewal.

Terminally differentiated absorptive colonocytes produce $I h h$ mRNA and Ihh protein (Fig. 1a-f). The epithelium of $I h h^{+/+}$mouse embryos consists of a monolayer of polarized epithelial cells that is organized into crypts at 16.5 days post coitum (d.p.c.; Fig. 1g,i). In contrast, the colonic epithelium of $\mathrm{Ihh}^{-/-}$embryos $^{3}$ is a multilayer and does not organize into crypts (Fig. $\mathbf{1 h}, \mathbf{j})$. Whereas some epithelial cells in the $\mathrm{Ih}^{+/+}$controls differentiate by 16.5 d.p.c. and are negative for the proliferation marker PCNA (Fig. 1k), all epithelial cells in $I h h^{-1-}$ colons are PCNA-positive at 16.5 d.p.c. (Fig. 11). These results indicate that Ihh may have a role in colonic epithelial differentiation.

As $\mathrm{Ihh}^{-1-}$ mice die before birth, we treated adult rats with the $\mathrm{Hh}$ signaling inhibitor cyclopamine to study the role of $\mathrm{Hh}$ signaling in adult colonic epithelial homeostasis. This plant steroidal alkaloid inhibits the cellular response to $\mathrm{Hh}$ signaling by directly binding to the Smo receptor ${ }^{4}$. The cyclopamine treatment induced a marked histo- logical change in the colonic enterocytes of all treated rats: the luminal end of the crypt had an enlarged nucleus and large cytoplasm in contrast to the slender nucleus and cytoplasm of terminally differentiated enterocytes of controls (Fig. 1 $\mathbf{m}-\mathbf{p}$ ). We used three molecular markers of colonic epithelial differentiation, Villin, a cytoskeletal protein that is specific for microvilli, the brush border enzyme carbonic anhydrase (CA) IV and the goblet cell marker intestinal trefoil factor (ITF), to characterize the maturation state of the enterocytes in the treated rats. Enterocytes in controls had a uniform Villin localization to the brush border (Fig. 1q). In contrast, the enterocytes in cyclopamine-treated rats showed heterogeneous Villin localization with loss of Villin expression at the brush border and redistribution of Villin to the cytoplasm (Fig. 1r). Whereas the colonic enterocytes of control rats highly expressed CA IV, the enterocytes in cyclopamine-treated rats were mostly CA IV-negative (Fig. 1s,t). In control rats, we found ITF staining mainly in goblet cells and on the brush border of normal enterocytes (Fig. 1u,w), as previously described by others ${ }^{5,6}$. Notably, the abnormal enterocytes in cyclopamine-treated rats showed strong immunoreactivity for ITF, indicating that they inappropriately express a marker of the goblet cell lineage (Fig. 1v, $\mathbf{x}$ ).

To confirm the role of Hh signaling in the maturation of colonic enterocytes, we used the HT-29 colon carcinoma differentiation model. HT-29 cells start to polarize and form apical microvilli when treated with the short-chain fatty acid butyrate ${ }^{7}$. We used two different markers of this differentiation process: Villin ${ }^{8}$ and the cell cycle inhibitor Cip-1 (also called p21 and Waf1), which has an essential role in growth inhibition during colonic epithelial differentiation ${ }^{9}$. Ihh protein was induced in HT-29 cells on treatment with butyrate (Fig. 2a). Treatment of HT-29 cultures with cyclopamine substantially reduced butyrate-mediated induction of Cip-1 and Villin (Fig. 2b,c). Expression of Cip-1 and Villin was induced with recombinant $\mathrm{N}$-terminal Shh peptide (Fig. 2d), which is $91 \%$ identical to the corresponding Ihh peptide, binds Ptc with the same affinity and induces the same biological response ${ }^{10}$. These

${ }^{1}$ Department of Experimental Internal Medicine and ${ }^{2}$ Department of Pathology, Academic Medical Center, Amsterdam, the Netherlands. ${ }^{3}$ Department of Pathology, Massachusetts General Hospital, Boston, Massachusetts, USA. ${ }^{4}$ Western Regional Research Center, ARS, USDA, Albany, California, USA. ${ }^{5}$ Department of Gastroenterology, Academic Medical Center, Amsterdam, the Netherlands. ${ }^{6}$ These authors contributed equally to this work. Correspondence should be addressed to G.R.v.d.B.(g.r.vandenbrink@amc.uva.nl) 
results indicate the Hh signaling is both necessary and sufficient for HT-29 cell differentiation. Thus, we concluded that Hh signaling regulates colonic epithelial differentiation in vivo and in vitro.

We examined the expression of several proteins that possibly interact with $\mathrm{Hh}$ signaling. The $\mathrm{Hh}$ binding receptor Ptc was broadly expressed in the epithelial cells along the crypt axis and on mesenchymal cells (Fig. 3a). Ihh may directly affect a wide range of target cells in both the epithelium and the mesenchyme. During morphogenesis of the fruit fly gut, the Wnt target gene Engrailed is important in the specification of the large intestine ${ }^{11}$. In the adult colon, Engrailed- 1 is expressed in epithelial cells along the entire crypt axis, with highest expression at its base (Fig. 3b). Engrailed-1 accumulated in the superficial enterocytes in cyclopamine-treated rats (Fig. 3c,d,i,j). The vertebrate Dpp homologs BMP2 and BMP4 are coexpressed with $H$ h genes during development ${ }^{12}$. We detected an epithelial gradient of BMP4 expression from the base to the tips of the crypts (Fig. 3e and ref. 1). In
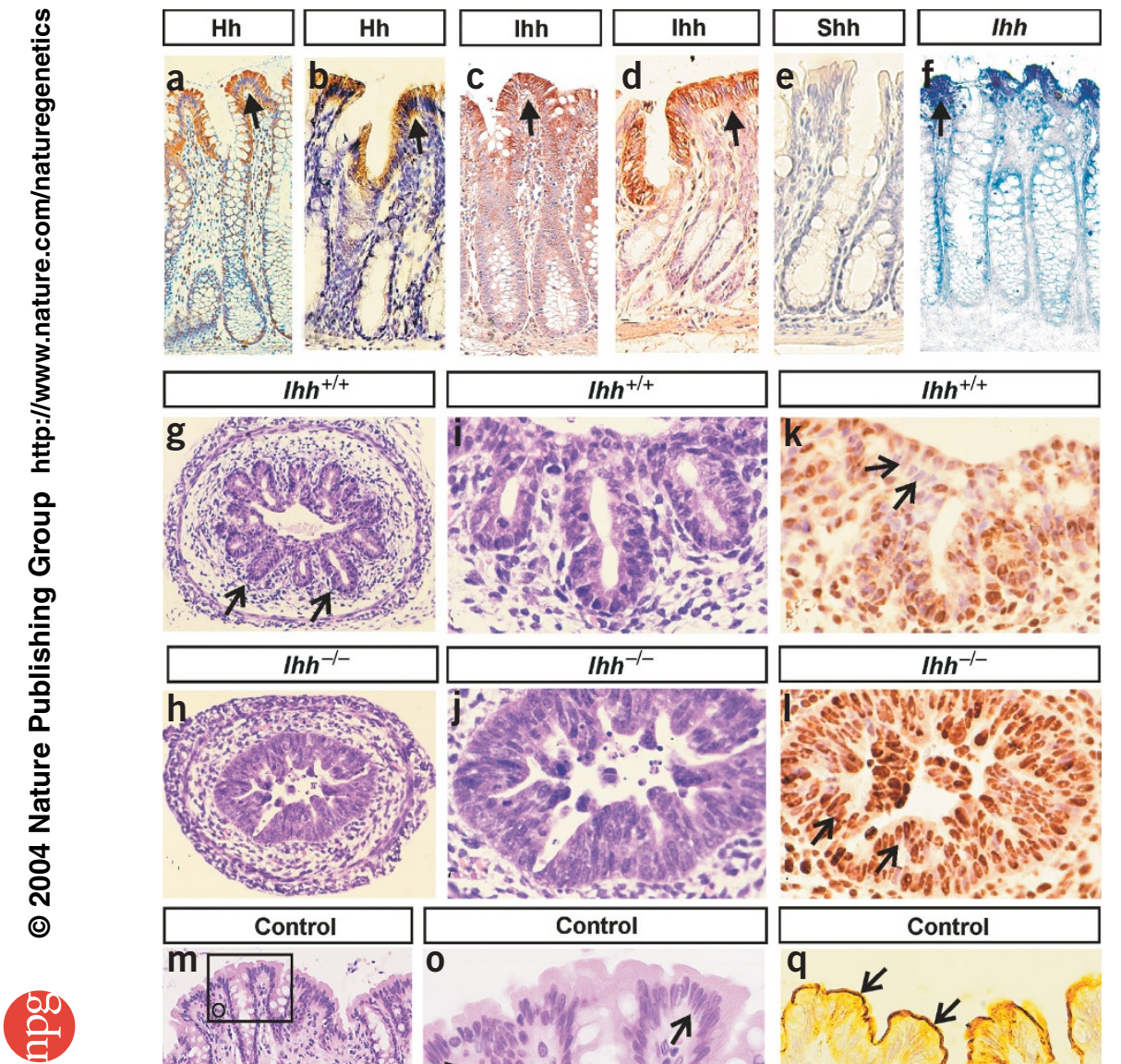
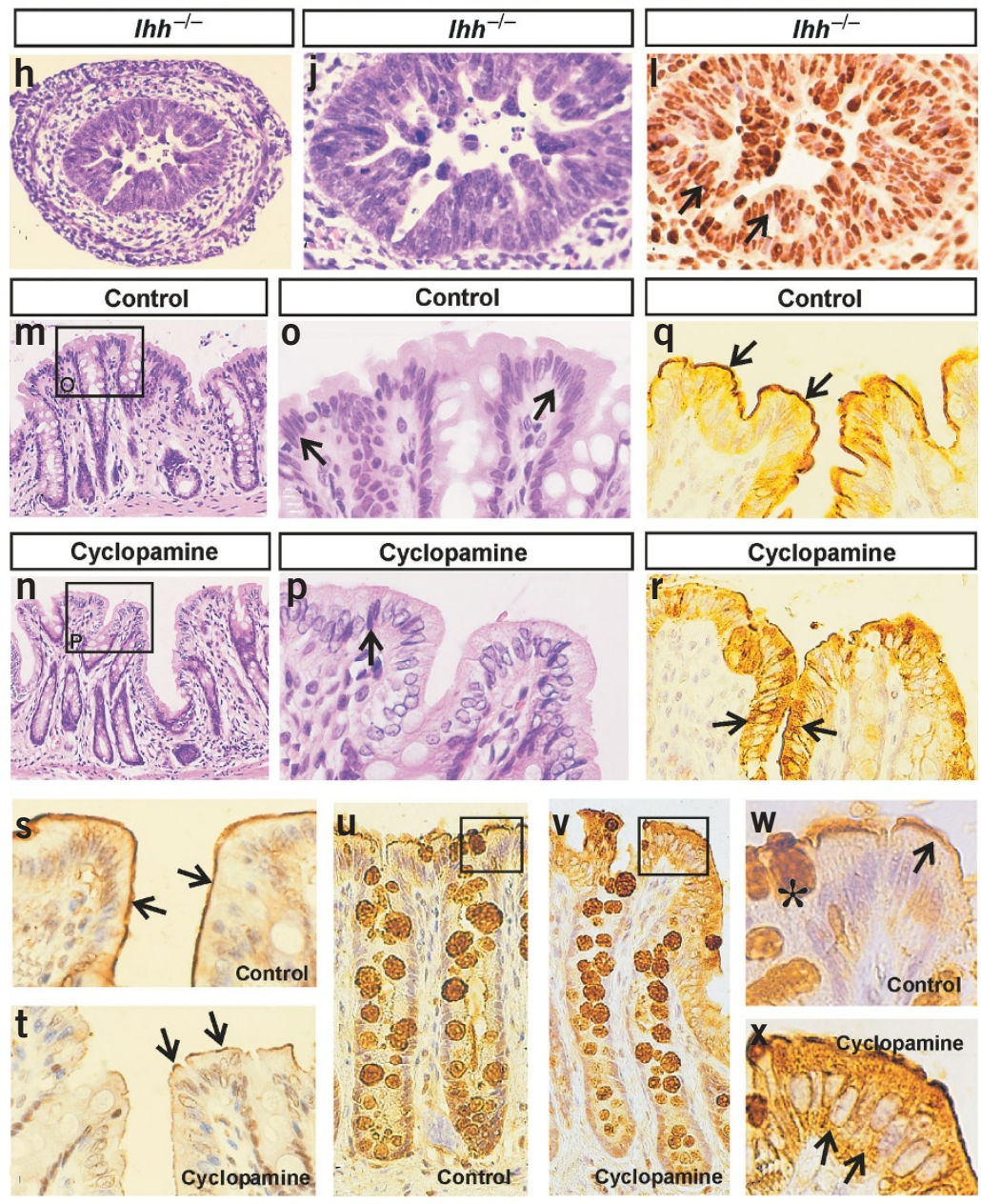

Figure 1 Hh signaling regulates colonic enterocyte differentiation in vivo.

$(\mathbf{a}, \mathbf{b})$ Immunohistochemical analysis of human (a) and rat (b) colon using an antibody (I-19) that reacts with all $\mathrm{Hh}$ proteins. Hh protein was expressed by the absorptive enterocytes in both species (arrows). (c,d) The Ihh specific antibody (C15) stained the same cells in both human (c) and rat (d) colon. (e) No staining was observed with antibodies to Shh (e) or to Dhh (data not shown; fetal testis used as positive control). (f) In situ hybridization with an Ihh probe on normal human colon. Terminally differentiated enterocytes (arrow) produced Ihh mRNA (these cells do not react with a Shh mRNA probe; see ref. 20). Colonic epithelial maturation was disturbed in the $/ \mathrm{hh}^{-1-}$ mouse

(129/BL6/CBA background, $n=6)$. (g-j) In colon of an control E16.5 embryo $(\mathbf{g}, \mathbf{i})$ the epithelial monolayer was organized into crypts (arrows in $\mathbf{g}$ ), whereas the epithelium of the $/ h^{-1-}$ mouse was multilayered and lacked crypts $(\mathbf{h}, \mathbf{j})$. (k) PCNA-positive cells were present mainly at the base of the crypts in control mice (I), whereas all epithelial cells were PCNA-positive in the $\mathrm{Ih}^{-1-}$ mouse. ( $\left.\mathbf{m}-\mathbf{x}\right)$ Cyclopamine treatment disturbed enterocyte maturation in adult rats. Hematoxylin and eosin staining of distal colon from control $(\mathbf{m}, \mathbf{0})$ and cyclopamine-treated (n,p) rats. (o) Arrows indicate normal, slender terminally differentiated enterocytes in controls. (p) Arrows indicate two enterocytes with normal appearance amid abnormal enterocytes with enlarged nuclei. (q,r) Villin immunohistochemistry. (q) Normal enterocytes showed light cytoplasmic staining and strong staining of the apical membrane (arrows). (r) In the abnormalappearing enterocytes of cyclopaminetreated rats, apical staining was diminished and cytoplasmic staining was enhanced (arrows). (s,t) CA IV immunohistochemistry. (s) Strong staining of the enterocyte apical membrane in control rats (arrows). (t) Loss of CA IV expression in the abnormal enterocytes in cyclopamine-treated rats. Arrows indicate a few remaining $C A$ IV-expressing enterocytes. (u-x) ITF immunohistochemistry. (w) Strong staining of goblet cells (asterisks) and apical staining of enterocytes (arrow) in control colon. (x) Strong immunoreactivity of the abnormal enterocytes (arrows) in cyclopamine-treated rats. 
a

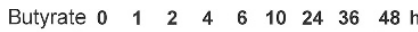

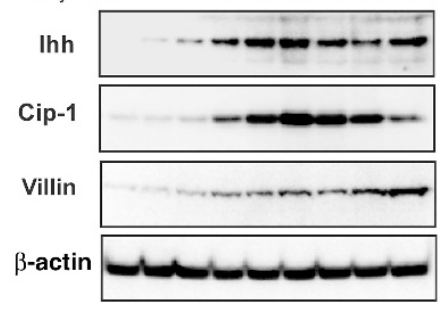

b

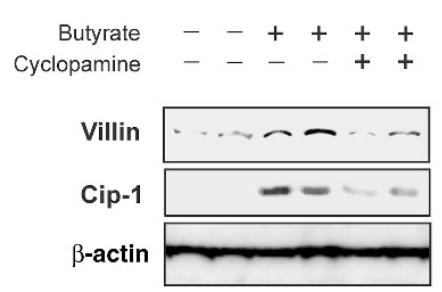

C

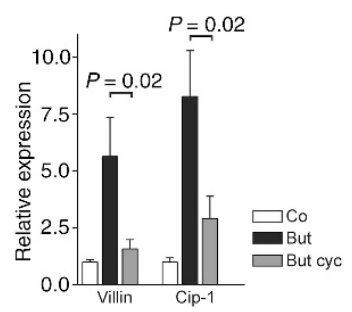

d

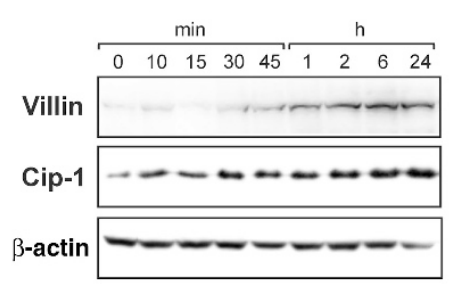

Figure $2 \mathrm{Hh}$ signaling regulates enterocyte differentiation in vitro. (a) Induction of Ihh expression on differentiation of HT-29 cells treated with $2.5 \mathrm{mM}$ butyrate for the indicated amounts of time. Immunoblots for Ihh (C-terminal antibody), Cip-1, Villin and $\beta$-actin. (b) Western blots of duplicate cultures of control HT-29 cells and HT-29 cells treated for $24 \mathrm{~h}$ with $2.5 \mathrm{mM}$ butyrate or with butyrate and cyclopamine (as indicated). (c) Mean \pm s.e.m. of three independent experiments (Co, control; But, butyrate; cyc, cyclopamine). (d) Western blots of HT-29 cells treated with $2.5 \mu \mathrm{ml}^{-1}$ recombinant Shh for the indicated amounts of time. Data are representative of at least three independent experiments.

the adult colon, BMP2 is expressed by the differentiated colonocytes ${ }^{13}$. Hh inhibition induced higher expression of BMP4 but had no effect on the expression of BMP2 (Fig. 3i,j). Immunohistochemistry showed loss of the BMP4 expression gradient in the epithelium of cyclopamine-treated rats (Fig. $\mathbf{3 e - j}$ ). Thus, we concluded that Hh signaling restricts expression of Engrailed-1 and BMP4 to the precursor cell compartment at the base of the colonic crypt.

Cyclin D1 was upregulated in cyclopamine-treated rats, and precursor cell proliferation increased 49\% (Fig. 3i,k).Engrailed-1, BMP4 and Cyclin D1 are established Wnt targets. Because they were all upregulated in response to cyclopamine treatment in vivo, Ihh signaling could negatively regulate $\beta$-catenin-TCF signaling and restrict it to the crypt. Using the TOPflash-FOPflash reporter assay for $\beta$-catenin-TCF signaling activity ${ }^{14}$, we showed that Ihh could completely abrogate endogenous $\beta$-catenin-TCF signaling in DLD- 1 colon cancer cells (Fig. 4a). This shutdown of $\beta$-catenin-TCF signaling correlated with downregulation of Wnt targets in DLD-1 cells (Fig. 4b). We observed no difference in the total cellular levels of $\beta$-catenin (Fig. 4b). However, we observed an almost complete downregulation of nuclear TCF4 expression and low levels of nuclear $\beta$-catenin in nuclear extracts of Ihh-transfected cells but not in control cultures transfected with a control vector (Fig. 4c). Thus, we conclude that Hh signaling restricts Wnt targets Engrailed-1 and BMP4 to the colonic precursor cell compartment in vivo and accordingly represses Wnt signaling in colon cancer cells in vitro.

Because Hh signaling negatively regulated Wnt signaling in the colonic epithelium, we were interested to see whether Wnt signaling affected the expression or activity of Ihh. Mutations in the gene APC initiate the development of colorectal cancer by constitutively activating the Wnt pathway. We examined Ihh expression in sporadic colonic adenomatous polyps and polyps of individuals with FAP (Fig. 5). In sporadic adenomatous polyps $(n=10)$, Ihh staining was lost from the
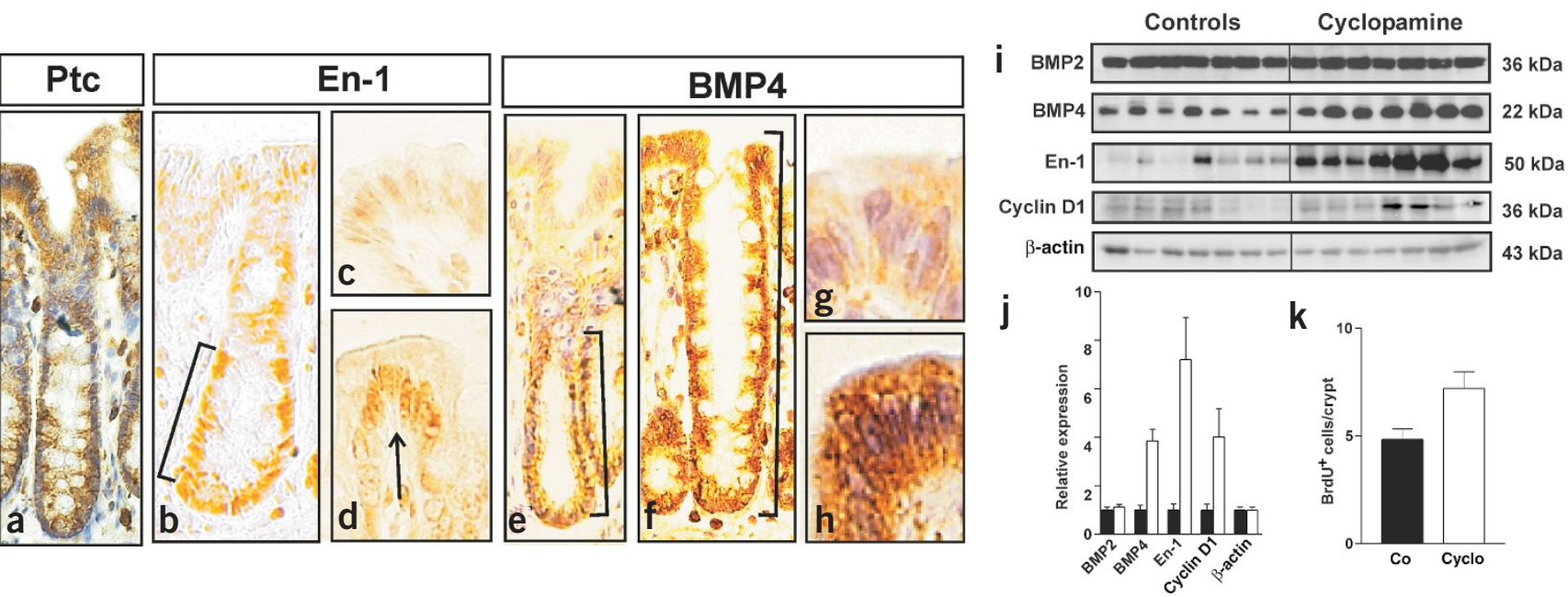

Figure $3 \mathrm{Hh}$ signaling restricts Wnt targets BMP4 and Engrailed-1 to the crypt base in the adult rat colon in vivo. (a) Ptc is expressed by epithelial cells throughout the crypt and in several stromal cell types (arrows). (b) A gradient of expression of Engrailed-1 (En-1) in epithelial cells. (c) In colon of control rats, engrailed-1 was expressed at low levels in superficial enterocytes. (d) Engrailed-1 accumulated in the superficial enterocytes in cyclopamine-treated rats (arrow). (e-g) The gradient of BMP4 expression from the base of the crypts to the superficial enterocytes in control rats $(\mathbf{e}, \mathbf{g}$ ) was lost in cyclopamine-treated rats (f,h). (i) Western blots. The first seven lanes contained colonic homogenates of seven individual control rats; the last seven lanes contained samples from cyclopamine-treated rats. En-1, Engrailed-1. (j) Quantification of blots shown in i. Mean \pm s.e.m. of the relative expression compared to the mean of the seven controls. $P$ values (Student's $t$-test): BMP2, $P=0.25$; BMP4, $P=0.0001$, Engrailed-1 (En-1), $P=0.002$, Cyclin D1, $P=0.01$. (k) The numbers of cells positive for 5 -bromodeoxyuridine (BrdU) per crypt was counted (mean of ten crypts) in cyclopamine-treated rats (Cyclo) and controls (Co; $P=0.01$ ). 

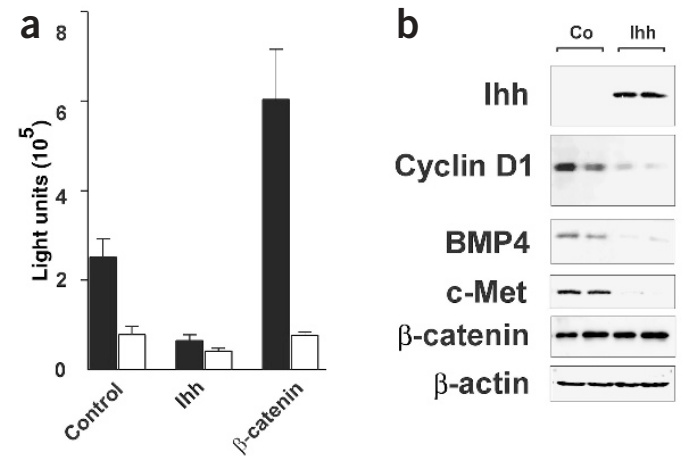

C

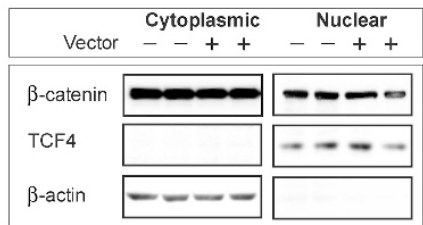

d

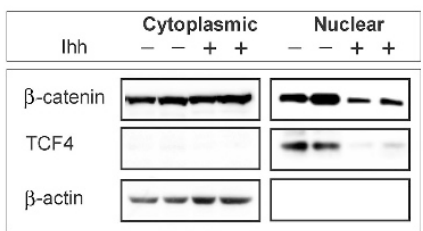

Figure 4 Ihh negatively regulates the Wnt pathway through downregulation of nuclear TCF4- $\beta$-catenin in vitro. (a) DLD-1 cells were transiently transfected with the TOPflash (filled bars) and FOPflash (open bars) reporter constructs in the presence or absence of an Ihh expression construct. A $\beta$-catenin expression construct was used as a positive control. Data are mean of three independent experiments $(n=12) ; P<0.001$ for TOP versus TOP+lhh. (b) Western blots showing the expression of Wnt targets (BMP4, Cyclin D1 and c-Met), $\beta$-catenin and $\beta$-actin (as a loading control) in duplicate cultures of DLD-1 cells transfected with either pcDNA3.1(-) (Co) or an Ihh expression construct and harvested $24 \mathrm{~h}$ after transfection. Data are representative of three independent experiments with duplicate cultures. (c) There were no changes in nuclear levels of either $\beta$-catenin or TCF4 in DLD- 1 cells transfected with control vector $(+)$ relative to untransfected cells $(-)$. (d) In nuclear extracts of cells transfected with Ihh (+), TCF4 disappeared almost completely and $\beta$ catenin was less abundant relative to untransfected cells (-). Data are representative of three independent experiments with duplicate cultures.

dysplastic epithelial cells (Fig. 5a-c). In contrast, we found normal Ihh staining in the morphologically normal tissue present in seven of these specimens and resection specimens of normal colonic tissue $(n=3)$. To study early lesions of the adenoma-carcinoma sequence, we examined 12 resection specimens of six different individuals with FAP. Multiple very small adenomas can be found in these specimens owing to the frequent inactivation of both $A P C$ alleles in the affected individuals. As in sporadic adenomas, Ihh expression was lost in the dysplastic epithelial cells in adenomas of individuals with FAP (data not shown). Notably, in two different individuals with FAP we found areas with complete loss of
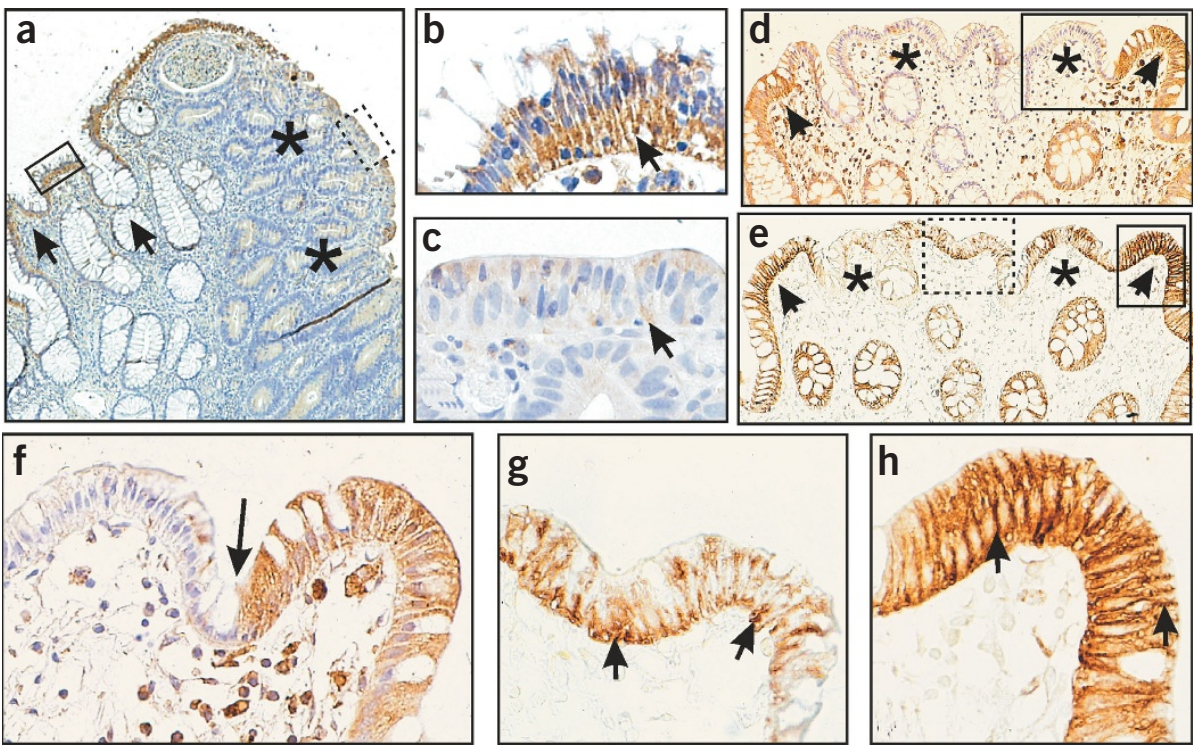

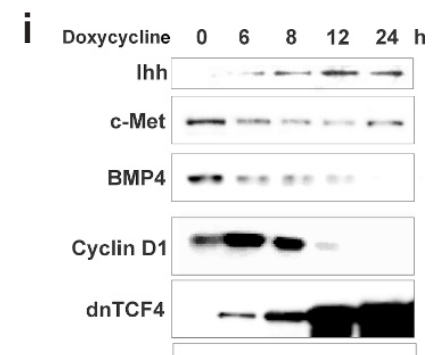

$\beta$-actin $--\infty-\infty$

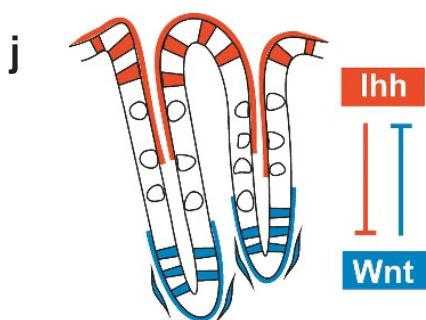

Figure 5 Loss of Ihh expression precedes the development of dysplasia in colon carcinogenesis. (a) An adenoma (asterisks) bordering histologically normal epithelium (arrows). (b) Histologically normal epithelial cells expressed Ihh. (c) Ihh was not expressed in the superficial dysplastic epithelial cells of the adenoma. (d-h) Immunohistochemical detection of Ihh and $\beta$-catenin in adjacent sections of tissue from an individual with FAP. (d,f) Ihh staining. Expression of Ihh was lost in a few morphologically normal crypts (asterisks), with a sharp transition to normal Ihh expression in the adjacent crypts on both sides (arrows). (e,g,h) $\beta$-catenin stain. The same crypts showed abnormal localization of $\beta$-catenin (f-h). (i) Western blots of DLD-1 cells carrying a doxycyclineinducible dnTCF4 construct. On treatment with doxycycline, FLAG epitope-tagged dnTCF4 was induced. An induction of Ihh expression was observed, whereas Wnt targets c-Met, BMP4 and Cyclin D1 (used as positive control) were downregulated. Data are representative of three independent experiments. (j) A model of the proposed Hh-Wnt axis in the colonic crypt. At the base of the crypt, Wnt factors are produced (probably by mesenchymal cell types ${ }^{1}$ ) that drive $\beta$-catenin-TCF signaling in epithelial precursor cells. Ihh expression is negatively regulated by $\beta$-catenin-TCF signaling and thus lost on mutation of $A P C$ during colorectal carcinogenesis. Ihh is expressed by mature colonocytes and regulates their differentiation. Hh signaling represses $\beta$-catenin-TCF signaling and restricts the expression of Wnt targets to the base of the colonic crypt. 
Ihh staining in the epithelium of a few morphologically normal adjacent crypts (Fig. 5d,f). $\beta$-catenin staining of consecutive sections (Fig. $\mathbf{5 e , g , h )}$ showed loss of membrane staining and accumulation of $\beta$-catenin precipitates at the basolateral membrane in the same area. This aberrant localization of $\beta$-catenin corresponds to that previously described in human aberrant crypt foci, the earliest morphologically recognizable lesions of $A P C$ mutants ${ }^{15}$. These results suggest that Ihh expression is downregulated in response to constitutive $\beta$-catenin-TCF signaling. We therefore examined Ihh expression in APC mutant DLD-1 cells that carry doxycycline-inducible dnTCF4 (ref. 1). We observed rapid induction of Ihh expression on inhibition of $\beta$-catenin-TCF signaling in these colon cancer cells (Fig. 5i), indicating that Ihh expression was negatively regulated by $\beta$-catenin-TCF signaling and that the APC mutation caused loss of Ihh expression in colonic polyps.

Blocking Hh signaling increased colonic epithelial cell proliferation, in contrast with the recent observation that cell growth of upper digestive tract tumors depends on Hh signaling ${ }^{16,17}$. Instead of acting on precursor cells, Hh signaling in the adult colon seems to act mainly on differentiating enterocytes, and the effects we observed on precursor cell proliferation may therefore be indirect. Our data corroborate previous findings ${ }^{17}$ that there was no Hh pathway activity in colon cancer cell lines. Our studies suggest that Hh pathway activity is lost from colon cancer cells owing to the activation of Wnt signaling in these cells. Notably, the redistribution of Villin from the apical membrane to the cytoplasm, loss of expression of brush border enzymes and inappropriate induction of ITF expression in the enterocyte lineage that we observed in cyclopamine-treated rats are all aspects of loss of differentiation that are also observed during colorectal carcinogenesis ${ }^{6,18}$. Thus, the loss of Ihh expression secondary to the Wnt-activating APC mutation may have a role in the development of dysplasia in response to the $A P C$ mutation.

Taken together, our in vivo and in vitro data suggest that Ihh is an important extrinsic signal for a differentiating colonic enterocyte and that it restricts $\beta$-catenin-TCF signaling to the precursor cell compartment at the base of the colonic crypt. We show that Ihh expression, in turn, is negatively regulated by Wnt signaling in vivo and in vitro.

\section{METHODS}

Antibodies. We used the following antibodies, with dilutions for immunohistochemistry shown first, followed by those used for immunoblotting. Antibodies to BMP2 (clone 355; 1:1,000; 1:2,000) and to BMP4 (clone 757; 1:500; 1:2,000) were from R\&D Systems. Antibodies to Shh, which recognizes the Shh precursor protein ${ }^{19}(\mathrm{~N}-19 ; 1: 200 ; 1: 2,000)$, to Hh (I-19; 1:50; 1:500), to Ihh (C-15; 1:50, 1:500), to Ptc (C-20, 1:50 for immunohistochemistry), to Villin (C-19; 1:1,000 for immunoblotting) and to $\beta$-actin (I-19; 1:5,000 for immunoblotting) were all from Santa Cruz. Antibody to Dhh (AVARP 7048, 1:50 for immunohistochemistry) was from Aviva. Antibody to Engrailed-1, developed in T.M. Jessell's laboratory (Columbia University, New York, New York, USA), was obtained from the Developmental Studies Hybridoma Bank. Antibodies to $\beta$-catenin (clone 14, 1:1,000 for immunohistochemistry) and to Cip-1 (clone 70, 1:5,000 for immunoblotting) were from Transduction Laboratories. Antibody to Cyclin D1 (clone DCS6, 1:1,000 for immunoblotting) was from Neomarkers. Antibodies to c-Met (clone DO-24, 1:2,000 for immunoblotting) and to TCF4 (clone 6H5-3, 1:500 for immunoblotting) were from Upstate Biotechnology. Rabbit polyclonal antibodies to Ptc (1:200 for immunohistochemistry), to ITF (1:500 for immunohistochemistry) and to CA IV (1:2,000 for immunohistochemistry) were gifts from R. Töftgard (Karolinska Institute, Sweden), D.K. Podolsky (Massachusetts General Hospital, Boston, Massachusetts, USA) and A. Waheed (St. Louis University School of Medicine, St. Louis, Missouri, USA), respectively. We confirmed the specificity of all antibodies used in immunohistochemistry on immunoblot and in experiments using the appropriate Ig controls. Secondary antibodies used were all from Dako.
Immunohistochemistry and in situ hybridization. We obtained formalin-fixed paraffin-embedded human resection specimens of normal colonic mucosa, sporadic adenomas and individuals with FAP from the archives of the pathology department of the Academic Medical Center. We carried out immunohistochemical analysis as described ${ }^{19}$. We carried out control immunohistochemical experiments leaving out the primary antibody or with control Ig. We carried out in situ hybridization on paraffin sections as described ${ }^{20}$ using digoxigenin-labeled mRNA probes for human $I H H$ and $S H H$, a gift from C. J. Tabin (Harvard Medical School, Boston, Massachusetts, USA).

Immunoblot. We dissected the distal half of the rat colon along the longitudinal axis and homogenized and processed one half for western blotting as described ${ }^{19}$.

Cyclopamine treatment. We treated 8-week-old female Wistar rats $(n=7)$ with daily intraperitoneal injections of $1 \mathrm{mg}$ per $\mathrm{kg}$ body weight of the Hh inhibitor cyclopamine complexed with 2-hydroxypropyl- $\beta$-cyclodextrin (Sigma) for $14 \mathrm{~d}$ as described ${ }^{19}$. Control rats $(n=7)$ received solvent only. The experiment was approved by the animal ethics review board of the University of Amsterdam.

Cell culture. We cultured HT 29 and DLD-1 colon cancer cell lines in the presence of $10 \%$ fetal calf serum and $4.5 \mathrm{mM}$ glucose (GIBCO). The TOPflashFOPflash TCF reporter constructs, a $\beta$-catenin expression construct and DLD-1 cells carrying a doxycycline-inducible dnTCF4 (cultured as described $^{21}$ ) were gifts from $\mathrm{H}$. Clevers (Hubrecht Laboratorium, the Netherlands). We transiently transfected DLD-1 cells with lipofectamine plus reagent (Invitrogen) according to the manufacturer's instructions. Luciferase activity was determined $24 \mathrm{~h}$ after transfection with a TOPCount microplate scintillation counter (Packard). The full-length Ihh expression construct was a gift from M. Hammerschmidt (Max-Planck Institute for Immunobiology, Germany). We carried out HT-29 cell experiments in six-well plates at the moment the cells reached confluence. We used butyrate (Sigma) at the indicated concentration and cyclopamine at $2 \mu \mathrm{g} \mathrm{ml}^{-1}$. To isolate nuclear extracts, we washed cells in ice-cold phosphate-buffered saline and scraped them into $200 \mu \mathrm{l}$ of ice-cold lysis buffer (20 mM HEPES, pH 7.9; $1.5 \mathrm{mM} \mathrm{MgCl}_{2} ; 10 \mathrm{mM}$ $\mathrm{KCl} ; 0.5$ dithiothreitol; $0.5 \mathrm{mM}$ Pefabloc, $0.5 \mu \mathrm{g} \mathrm{ml}^{-1}$ leupeptin; $0.5 \mu \mathrm{g} \mathrm{ml}^{-1}$ aprotinin). We passed the lysate through a 27-gauge needle ten times. After centrifugation at $600 \mathrm{~g}$, we washed the resultant pellets (nuclear fraction) again in lysis buffer and resuspended them in sample buffer. We diluted the supernatants in sample buffer to yield the cytosolic fraction.

\section{ACKNOWLEDGMENTS}

We thank H. Clevers, M. Hammerschmidt, C. Tabin, D.K Podolsky, A. Waheed and R. Töftgard for reagents; A. Vortkamp for colons of $I h h^{-/-}$mice; and J. Daalhuisen for technical assistance.

\section{COMPETING INTERESTS STATEMENT}

The authors declare that they have no competing financial interests.

Received 8 December 2003; accepted 21 January 2004 Published online at http://www.nature.com/naturegenetics/

1. van de Wetering, M. et al. The beta-Catenin/TCF-4 complex imposes a crypt progenitor phenotype on colorectal cancer cells. Cell 111, 241-250 (2002).

2. Bienz, M. \& Clevers, H. Linking colorectal cancer to Wnt signaling. Cell 103 311-320 (2000)

3. St-Jacques, B., Hammerschmidt, M. \& McMahon, A.P. Indian hedgehog signaling regulates proliferation and differentiation of chondrocytes and is essential for bone formation. Genes Dev. 13, 2072-2086 (1999).

4. Chen, J.K., Taipale, J., Cooper, M.K. \& Beachy, P.A. Inhibition of Hedgehog signaling by direct binding of cyclopamine to Smoothened. Genes Dev. 16, 2743-2748 (2002).

5. Suemori, S., Lynch-Devaney, K. \& Podolsky, D.K. Identification and characterization of rat intestinal trefoil factor: tissue- and cell-specific member of the trefoil protein family. Proc. Natl. Acad. Sci. USA 88, 11017-11021 (1991).

6. Taupin, D., Ooi, K., Yeomans, N. \& Giraud, A. Conserved expression of intestinal trefoil factor in the human colonic adenoma-carcinoma sequence. Lab. Invest. 75 , 25-32 (1996).

7. Augeron, C. \& Laboisse, C.L. Emergence of permanently differentiated cell clones in a human colonic cancer cell line in culture after treatment with sodium butyrate. Cancer Res. 44, 3961-3969 (1984).

8. Pringault, E., Arpin, M., Garcia, A., Finidori, J. \& Louvard, D. A human villin cDNA clone to investigate the differentiation of intestinal and kidney cells in vivo and in culture. EMBO J. 5, 3119-3124 (1986). 


\section{LETTERS}

9. Archer, S.Y., Meng, S., Shei, A. \& Hodin, R.A. p21(WAF1) is required for butyratemediated growth inhibition of human colon cancer cells. Proc. Natl. Acad. Sci. USA 95, 6791-6796 (1998).

10. Pathi, S. et al. Comparative biological responses to human Sonic, Indian, and Desert hedgehog. Mech. Dev. 106, 107-117 (2001).

11. Lengyel, J.A. \& Iwaki, D.D. It takes guts: the Drosophila hindgut as a model system for organogenesis. Dev. Biol. 243, 1-19 (2002).

12. Bitgood, M.J. \& McMahon, A.P. Hedgehog and Bmp genes are coexpressed at many diverse sites of cell-cell interaction in the mouse embryo. Dev. Biol. 172, 126-138 (1995).

13. Hardwick, J.C. et al. Bone morphogenetic protein 2 is expressed by, and acts upon, mature epithelial cells in the colon. Gastroenterology 126, 111-121 (2004).

14. Korinek, V. et al. Constitutive transcriptional activation by a beta-catenin-Tcf complex in APC-/- colon carcinoma. Science 275, 1784-1787 (1997).

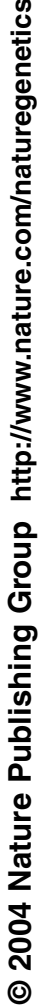

15. Hao, X.P., Pretlow, T.G., Rao, J.S. \& Pretlow, T.P. Beta-catenin expression is altered in human colonic aberrant crypt foci. Cancer Res. 61, 8085-8088 (2001)

16. Thayer, S.P. et al. Hedgehog is an early and late mediator of pancreatic cancer tumorigenesis. Nature 425, 851-856 (2003).

17. Berman, D.M. et al. Widespread requirement for Hedgehog ligand stimulation in growth of digestive tract tumours. Nature 425, 846-851 (2003)

18. West, A.B et al. Localization of villin, a cytoskeletal protein specific to microvilli, in human ileum and colon and in colonic neoplasms. Gastroenterology 94, 343-352 (1988)

19. van den Brink, G.R. et al. Sonic hedgehog regulates gastric gland morphogenesis in man and mouse. Gastroenterology 121, 317-328 (2001).

20. van den Brink, G.R. et al. Sonic hedgehog expression correlates with fundic gland differentiation in the adult gastrointestinal tract. Gut 51, 628-633 (2002).

21. Boon, E.M., van der Neut, R., van de Wetering, M., Clevers, H. \& Pals, S.T. Wnt signaling regulates expression of the receptor tyrosine kinase met in colorectal cancer. Cancer Res. 62, 5126-5128 (2002). 